INPLASY

PROTOCOL

To cite: Shi et al. Diagnostic value of ultrasound combined with MRI in cholecystolithiasis: a protocol for systematic review and meta analysis. Inplasy protocol 202130003. doi:

10.37766/inplasy2021.3.0003

Received: 02 March 2021

Published: 02 March 2021

Corresponding author:

Yu Shi

zero623996111@163.com

Author Affiliation:

Chongqing Beibei Traditional

Chinese Medical Hospital,

China

Support: Objective study of TCM.

Review Stage at time of this submission: The review has not yet started.

Conflicts of interest:

None declared.

\section{Diagnostic value of ultrasound combined with MRI in cholecystolithiasis: a protocol for systematic review and meta analysis}

Shi, Y1; Yang, MF².

Review question / Objective: Do ultrasound combined with MRI have diagnostic value in cholecystolithiasis.

Condition being studied: Cholecystitis (ko-luh-sis-TIE-tis) is inflammation of the gallbladder.If left untreated, cholecystitis can lead to serious, sometimes life-threatening complications, such as a gallbladder rupture. Abdominal ultrasound, endoscopic ultrasound, or a computerized tomography (CT) scan can be used to create pictures of the gallbladder that may reveal signs of cholecystitis or stones in the bile ducts and gallbladder. Many studies have investigated the diagnostic value of ultrasound combined with MRI in cholecystolithiasis, but the evidence has been insufficient to apply these modalities when diagnosing new patients.

INPLASY registration number: This protocol was registered with the International Platform of Registered Systematic Review and Meta-Analysis Protocols (INPLASY) on 02 March 2021 and was last updated on 22 April 2021 (registration number INPLASY202130003).

\section{INTRODUCTION}

Review question / Objective: Do ultrasound combined with MRI have diagnostic value in cholecystolithiasis.
Condition being studied: Cholecystitis (koluh-sis-TIE-tis) is inflammation of the gallbladder.If left untreated, cholecystitis can lead to serious, sometimes lifethreatening complications, such as a gallbladder rupture.Abdominal ultrasound, endoscopic ultrasound, or a computerized 
tomography (CT) scan can be used to create pictures of the gallbladder that may reveal signs of cholecystitis or stones in the bile ducts and gallbladder.Many studies have investigated the diagnostic value of ultrasound combined with MRI in cholecystolithiasis, but the evidence has been insufficient to apply these modalities when diagnosing new patients.

\section{METHODS}

Participant or population: Participants were patients with a clear diagnosis of Cholecystitis, regardless of the age, gender and severity.

Intervention: Ultrasound combined with MRI.

\section{Comparator: Conventional exams.}

Study designs to be included: Diagnostic trials with 2-by-2 tabulated data including true positive (TP), false positive (FP), true negative (TN) and false negative (FN) rates that could be extracted directly or indirectly.

Eligibility criteria: A clear diagnosis of Cholecystitis, regardless of the age, gender and severity.

Information sources: We will search the following sources for the identification of trials: The Cochrane Library, PubMed, EMBASE, Chinese Biomedical Literature Database (CBM), Chinese National Knowledge Infrastructure Database(CNKI), Chinese Science and Technique Journals Database (VIP), and the Wanfang Database. The searches were limited to articles published in 2020, but no language restrictions were imposed.

\section{Main outcome(s): Diagnostic accuracy.}

Additional outcome(s): Binary variable is analysed using odds ratio (OR) with $95 \%$ confidence intervals (Cls).

Quality assessment / Risk of bias analysis: The Cochrane Collaboration's risk of bias tool will be employed to evaluate the methodological quality of eligible RCTs. The tool focuses on each study's selection bias (random sequence generation and allocation concealment), performance bias (blinding of participants and personnel), detection bias (blinding of outcome assessment), attrition bias (incomplete outcome data), selective reporting bias and other bias. Two independent reviewers will evaluate the quality of RCTs with this tool. Any discrepancies between the two reviewers will be settled through discussion or the introduction of a third reviewer.

Strategy of data synthesis: The data for statistical analysis will be extracted into an excel file. Dichotomous data will be investigated by using a risk ratio with $95 \%$ Cls. For continuous outcomes, data will be analyzed by using a standard mean difference (SMD) with $95 \% \mathrm{Cls}$ or a weighted mean difference (WMD). The WMD will be used for the same scale or the same assessment instrument; SMD will be used for different assessment tools. A network meta-analysis based on frequentist framework will be conducted by using the net-meta package in $R$ software (www.r-project.org, version 3.2.0), integrating direct and indirect evidences of included RCTs. A network plot will be made to show the number and interrelations of interventions and direct comparison between interventions. The interventions results will be ranked based on their $P$ values. Consistency will be assessed by implementing Cochran's $Q$ statistics, and if with obvious heterogeneity, a metaregression process will be performed to determine its potential source with a netheat plot.

Subgroup analysis: A subgroup analysis will be performed to determine the potential heterogeneity and inconsistency clinically and statistically. This include age, gender and disease duration of patients, trial blinding, evidence quality and so on. Meta-regression analysis will be implemented to quantify the inter-subgroup difference and explore statistical significance. 
Sensitivity analysis: We will carry out a sensitivity analysis by systematically removing every study and checking the pooled results for the remaining studies to see if there is any significant change in test performance.

Country(ies) involved: China.

Keywords: MRI, ultrasound.

Contributions of each author:

Author 1 - Yu Shi.

Email: zero623996111@163.com

Author 2 - Mingfang Yang.

Email: 615558624@qq.com 